\title{
Left Atrial scar assessment using imaging with isotropic spatial resolution and compressed sensing
}

\author{
Mehmet Akcakaya ${ }^{1 *}$, Susie Hong ${ }^{1}$, Raymond H Chan ${ }^{1}$, Tamer A Basha ${ }^{1}$, Mehdi H Moghari ${ }^{1}$, Kraig V Kissinger ${ }^{1}$, \\ Beth Goddu', Mark E Josephson ${ }^{1}$, Warren J Manning ${ }^{1,2}$, Reza Nezafat ${ }^{1}$
}

From 15th Annual SCMR Scientific Sessions

Orlando, FL, USA. 2-5 February 2012

\section{Summary}

We assess left atrial scar using late gadolinium enhancement (LGE) with isotropic spatial resolution of $1.4 \mathrm{~mm}^{3}$ by using highly accelerated LOST [1] reconstruction.

\section{Background}

Atrial fibrillation (AF) is the most common sustained cardiac arrhythmia [2]. Pulmonary vein isolation (PVI) using radiofrequency $(\mathrm{RF})$-ablation is the leading treatment for AF. Recently, LGE imaging of the LA has been used to identify atrial wall scar due to RF-ablation [3]. However, current LGE methods have limited spatial resolution that substantially impact assessment of scar in the complex geometry of PVs and LA. In this study, we sought to utilize prospective random k-space sampling and LOST [1] for accelerated LGE imaging, where reduction in imaging time was traded-off for improved isotropic spatial-resolution.

\section{Methods}

23 patients with history of AF ( 6 females, $58.1 \pm 9.6$ years, 9 pre-PVI, 2 with history of PVI; 8 post-PVI; 3 with both pre and post-PVI) were recruited for this study. LGE images were acquired 10-to-20 minutes after bolus infusion of $0.2 \mathrm{mmol} / \mathrm{kg}$ Gd-DTPA. Free-breathing ECG-triggered navigator-gated inversion-recovery GRE sequences were used for all acquisitions (TR/TE/ $\alpha=5.2 /$ $2.6 \mathrm{~ms} / 25^{\circ}, \mathrm{FOV}=320 \times 320 \times 100 \mathrm{~mm}^{3}$ ). The PV inflow artifact reduction technique in [4] was also utilized. For each patient, a standard non-isotropic 3D LGE scan $\left(1.4 \times 1.4 \times 4.0 \mathrm{~mm}^{3}\right)$ and a 3 -fold-accelerated high-

${ }^{1}$ Medicine, Beth Israel Deaconess Medical Center, Harvard Medical School, Boston, MA, USA

Full list of author information is available at the end of the article resolution 3D LGE scan $\left(1.4^{3} \mathrm{~mm}^{3}\right)$ were performed, with randomized acquisition order. For random undersampling, central k-space $(45 \times 35$ in $\mathrm{ky}-\mathrm{kz})$ was fullysampled, edges randomly discarded, and phase reordering performed as in [5]. Acquisition times were $\sim 4$ mins assuming $100 \%$ scan-efficiency at $70 \mathrm{bpm}$ for both scans.

All undersampled data were reconstructed offline using LOST [1]. LOST-reconstructed high-resolution, and standard LGE images were scored by two blinded readers for diagnostic value, presence of LGE(yes/no); and image quality in axial( $\mathrm{Ax})$, coronal $(\mathrm{Co})$ and sagittal (Sa) views $(1=$ poor, $4=$ excellent $)$.

\section{Results}

Three cases were declared non-diagnostic due to contrast-washout and imperfect inversion-time. LGE was visually present in 14 of the remaining 20 patients based on standard-LGE images, and 12 based on LOST-reconstructed ones (disagreement on one pre- and one postPVI patient). Figure 1 and 2 show comparisons of isotropic vs. non-isotropic LGE images in two patients. Image scores for LOST-LGE: $\mathrm{Ax}=2.90 \pm 0.70, \mathrm{Sa}=3.33$ $\pm 0.66, \mathrm{Co}=3.00 \pm 0.63$; and standard LGE: $\mathrm{Ax}=3.76 \pm 0.54$, $\mathrm{Sa}=2.48 \pm 0.60, \mathrm{Co}=2.24 \pm 0.44$, where differences were significant in all views.

\section{Conclusions}

LOST allows isotropic spatial-resolution in LGE for assessment of LA and PV scar. Isotropic resolution allows reformatting LGE images in any orientation and facilitates assessment of scar. Further clinical study is needed to assess if the improved spatial resolution will impact the diagnostic interpretation of data. 

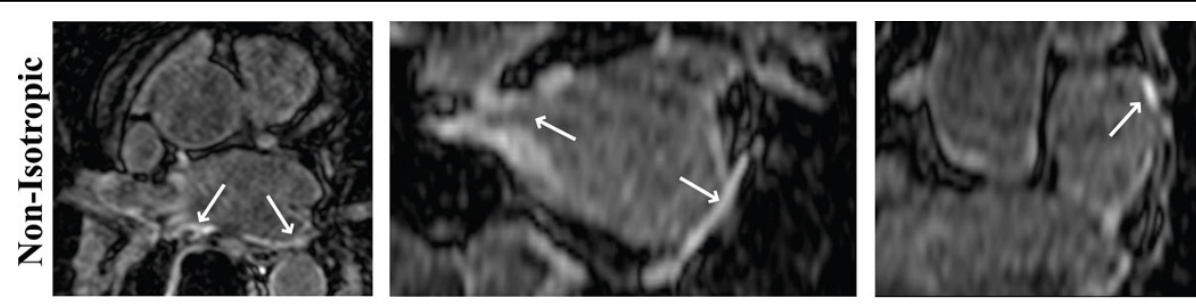

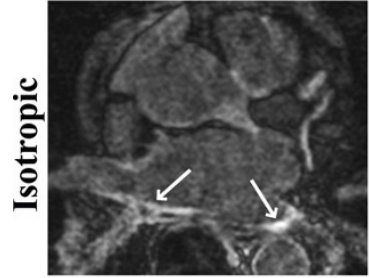

(a)

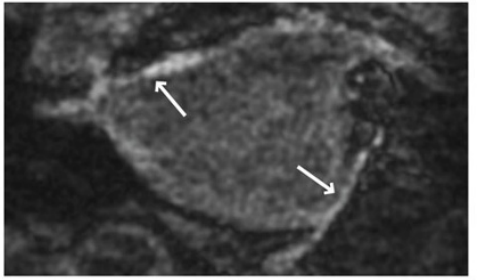

(b)

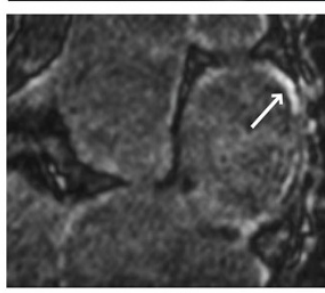

(c)

Figure 1 Axial (a), coronal (b) and reformatted (c) LA LGE images from a patient who underwent PVI. LOST-reconstructed images from the isotropic resolution scan with acceleration rate 3 enable better visualization from different orientations, whereas the standard non-isotropic LGE images suffer from blurring in sagittal and coronal views.

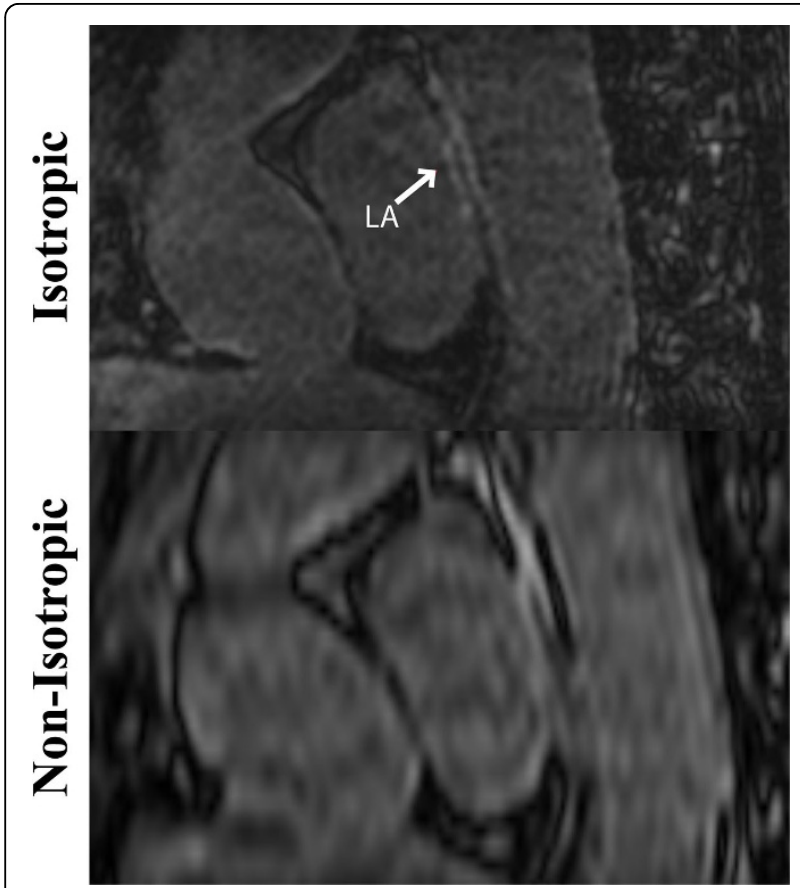

Figure $2 \mathrm{~A}$ sagittal view showing enhancement of the $L A$ and the descending aorta in a patient who underwent PVI. The isotropic resolution of the LOST-reconstructed images from the accelerated scan allows clear visualization of separate regions of enhancement, whereas the non-isotropic resolution exhibits blurring of these regions.

\section{Funding}

NIH R01EB008743-01A2.

\section{Author details}

'Medicine, Beth Israel Deaconess Medical Center, Harvard Medical School, Boston, MA, USA. ${ }^{2}$ Radiology, Beth Israel Deaconess Medical Center, Harvard Medical School, Boston, MA, USA.

Published: 1 February 2012

\section{References}

1. Akcakaya : MRM. 2011

2. Lloyd-Jones : Circulation. 2010.

3. Peters : Radiology. 2007.

4. Hedjazi : MRM. 2011

5. Basha : ISMRM. 2011.

\section{doi:10.1186/1532-429X-14-S1-O8}

Cite this article as: Akcakaya et al:: Left Atrial scar assessment using imaging with isotropic spatial resolution and compressed sensing. Journal of Cardiovascular Magnetic Resonance 2012 14(Suppl 1):O8.

\section{Submit your next manuscript to BioMed Central and take full advantage of:}

- Convenient online submission

- Thorough peer review

- No space constraints or color figure charges

- Immediate publication on acceptance

- Inclusion in PubMed, CAS, Scopus and Google Scholar

- Research which is freely available for redistribution 